\section{Clinical acceleration of JAK2 p.V617F driven myeloproliferative disease due to a new uncommon homozygous MPL p.Y591D mutation}

Three driver mutations (JAK2, CALR and MPL) comprise one of the major diagnostic criteria for Philadelphia negative $(P h-)$ myeloproliferative neoplasms $(\mathrm{MPN})$ in the current World Health Organisation (WHO) classification. ${ }^{1}$ Although driver mutations were previously thought to be mutually exclusive, recent reports have demonstrated their uncommon co-existence. ${ }^{2-4}$ The impact of single driver mutations on phenotype and prognosis has been established, though the clinical implication of coexisting driver mutations remains unclear. ${ }^{5-7}$ With more sensitive and cost-effective next generation sequencing (NGS) applications for patients with blood cancers, novel mutations are being discovered that have important clinical and prognostic significance. Herein, we report a case of three stages of molecular and clinical evolution of a MPN from polycythemia vera (PV) to post-PV myelofibrosis (MF), and then to accelerated phase MF. We found the acquisition of new mutations and copy number changes using an NGS panel that correlated with changes in clinical features; the late acquisition of a new MPL p.Y591D mutation in addition to baseline JAK2 p.V617F and $A S X L 1$ p.R1415Ter mutations resulted in accelerated disease with severe leukocytosis.

Our case is of a 73-year-old woman who was diagnosed with JAK2 p.V617F mutant PV in 2008. The initial diagnosis of PV was made based on WHO criteria, including a positive test for the JAK2 p.V617F mutation and a low serum erythropoietin (EPO) level (2 IU/mL); a bone marrow biopsy was not performed. Initial treatment included hydroxycarbamide, aspirin and intermittent venesections. Retrospective molecular assessment using a custom designed AmpliSeq panel ${ }^{8}$ run on an Illumina MiniSeq analyser showed heterozygosity for the JAK2 p.V617F mutation (variant allele frequency $[\mathrm{VAF}]=0.51)$ and a heterozygous single nucleotide variant (SNV) in the ASXL1 gene, leading to a nonsense amino acid change, p.R1415Ter (VAF=0.43) (Table 1). This variant has been reported as a pathogenic mutation in the Catalogue of Somatic Mutations in Cancer (COSMIC) database (v90). ${ }^{9}$

Six years after diagnosis, the patient developed new fatigue, night sweats and splenomegaly. Full blood examination (FBE) showed anemia (hemoglobin 9.1g/dL), leucocytosis (white cell count [WCC] $17 \times 10^{9} / \mathrm{L}$ ) (Figure 1) and thrombocytosis (platelets $661 \times 10^{\circ} / \mathrm{L}$ ) (Figure 2, blue arrow). There was also new monocytosis, basophilia and eosinophila in the blood (Figure 1, blue arrow). The blood smear showed leucoerythroblastosis with prominent teardrop poikilocytes. The lactate dehydrogenase was $1049 \mathrm{U} / \mathrm{L}$ (reference range: 125-255 U/L). A bone marrow biopsy demonstrated a markedly hypercellular and fibrotic marrow with increased granulocytic proliferation and abnormal megakaryocyte clustering, consistent with post-PV MF. Molecular analysis of DNA extracted from the trephine detected the same two sequence variants; i.e., (i) a $J A K 2$ p.V617F missense mutation with a VAF of 0.82 suggesting uniparental disomy (UPD) in a fraction of the cells of the malignant clone, and (ii) the same ASXL1 mutation at the same VAF of 0.45 (Table 1) (Figure 3 ). No other variants were detected in the coding regions of 86 genes that includes most variants documented to be associated with progression of PV to MF.,

Hydroxycarbamide was changed to ruxolitinib $15 \mathrm{mg}$ twice daily (Figure 2) with improvement in constitutional symptoms and reduction in spleen size. The FBE remained stable with a mild anemia (hemoglobin 9.5-11 $\mathrm{g} / \mathrm{dL}$ ), moderate leucocytosis white cell count (WCC) 25 $35 \times 10^{9} / \mathrm{L}$ ) and mild thrombocytopenia (platelets 75 $\left.125 \times 10^{9} / \mathrm{L}\right)$. Four years after commencing ruxolinitib, there was sudden progression into an accelerated phase of disease with marked leucocytosis (WCC $115 \times 10^{9} / \mathrm{L}$ ) (Figure 1, red arrow) and splenomegaly. The hemoglobin and platelet values (Figure 2) remained stable. A blood smear showed prominence of myelocytes and basophils. A repeat bone marrow trephine was fibrotic with increased abnormal megakaryocytes, but not markedly different from the bone marrow performed four years earlier. A $B C R-A B L 1$ fusion transcript was not detected by quantitative real-time PCR. Molecular analysis on the peripheral blood using the same NGS panel now detected three sequence variants: (i) the same JAK2 p.V617F missense mutation with a VAF $=0.97$, consistent with a selective advantage for the clone with UPD, (ii) the same $A S X L 1$ nonsense mutation with a $\mathrm{VAF}=0.48$, and (iii) a new SNV in the MPL gene, leading to a p.Y591D amino acid change (Table 1 and Figure 3 ). This is not one of the common $\mathrm{p}$.W515L/K/A gain of function mutations in $M P L$, but has been reported to be pathogenic. ${ }^{10}$ This new $M P L$ variant was present at a VAF $=0.96$, so we performed concurrent peripheral blood cytogenetic testing, with no abnormalities found. In particular, monosomy 1 , a common cytogenetic abnormality in $M P L$-driven MPN, was not present, so we conclude that there must be UPD

Table 1. Evolution of mutations and copy number variations at times of disease progression.

\begin{tabular}{|c|c|c|c|c|}
\hline Disease Stage & NGS Results & JAK2 & ASXL1 & MPL \\
\hline PV & Genotype & c. $1849 \mathrm{G}>\mathrm{T}$ & c. 4243 C>T & Wild-type* \\
\hline \multirow[t]{2}{*}{ (2008) } & Protein & p.V617F & p.R1415Ter & \\
\hline & Variant allele frequency & 0.51 & 0.43 & \\
\hline PV-MF & Genotype & c. $1849 \mathrm{G}>\mathrm{T}$ & c. 4243 C > T & Wild-type* \\
\hline \multirow[t]{2}{*}{ (2014) } & Protein & p.V617F & p.R1415Ter & \\
\hline & Variant allele frequency & 0.82 & 0.45 & \\
\hline Accelerated MF & Genotype & c. $1849 \mathrm{G}>\mathrm{T}$ & c. $4243 \mathrm{C}>\mathrm{T}$ & c. $1771 \mathrm{~T}>\mathrm{G}$ \\
\hline \multirow[t]{2}{*}{ (2019) } & Protein & p.V617F & p.R1415Ter & p.Y591D \\
\hline & Variant allele frequency & 0.97 & 0.48 & 0.96 \\
\hline
\end{tabular}

*: Limit of detection is $0.5 \%$ variant allele frequency; NGS: next generation sequencing; PV: polycythemia vera; MF: myelofibrosis; PV-MF: post-polycythemia myelofibrosis. 
in the region of the MPL mutation. This also occurs in MPL-driven MPN, and is under positive selection. ${ }^{10} \mathrm{At}$ this time point, no new additional variants, such as those in SRSF2, CBL, U2AF1, IDH1 and IDH2 that are characteristic of chronic myelomonocytic leukaemia, were detected. Thus, we are confident the leucocytosis and monocytosis are both likely due to the new MPL variant.

Hydroxycarbamide was added to ruxolitinib to control the leucocytosis and splenomegaly (Figure 2). The WCC returned to $18 \times 10^{9} / \mathrm{L}$, with an associated reduction in hemoglobin $(94 \mathrm{~g} / \mathrm{dL})$ and platelet count $\left(21 \times 10^{9} / \mathrm{L}\right)$. The patient remained essentially asymptomatic throughout the accelerated phase of disease. The blast cells remained $<1 \%$ of total WCC throughout.
This is a clear case of progression from PV to MF and then accelerated phase MF. The first clinical change (PV to $\mathrm{MF}$ ) was associated with new monocytosis, eosinophilia and basophilia. The clinical progression corresponded to UPD of the JAK2 p.V617F mutation, with no other mutations commonly associated with monocytosis or fibrosis detected by comprehensive NGS. Whole exome sequencing to detect rarer mutations was not performed.

The second clinical progression (MF to accelerated MF characterised by marked leukocytosis) was associated with acquisition of a new uncommon mutation in MPL. The VAF for the pathogenic ASXL1 mutation, p.R1415Ter, was unchanged from the original time point,

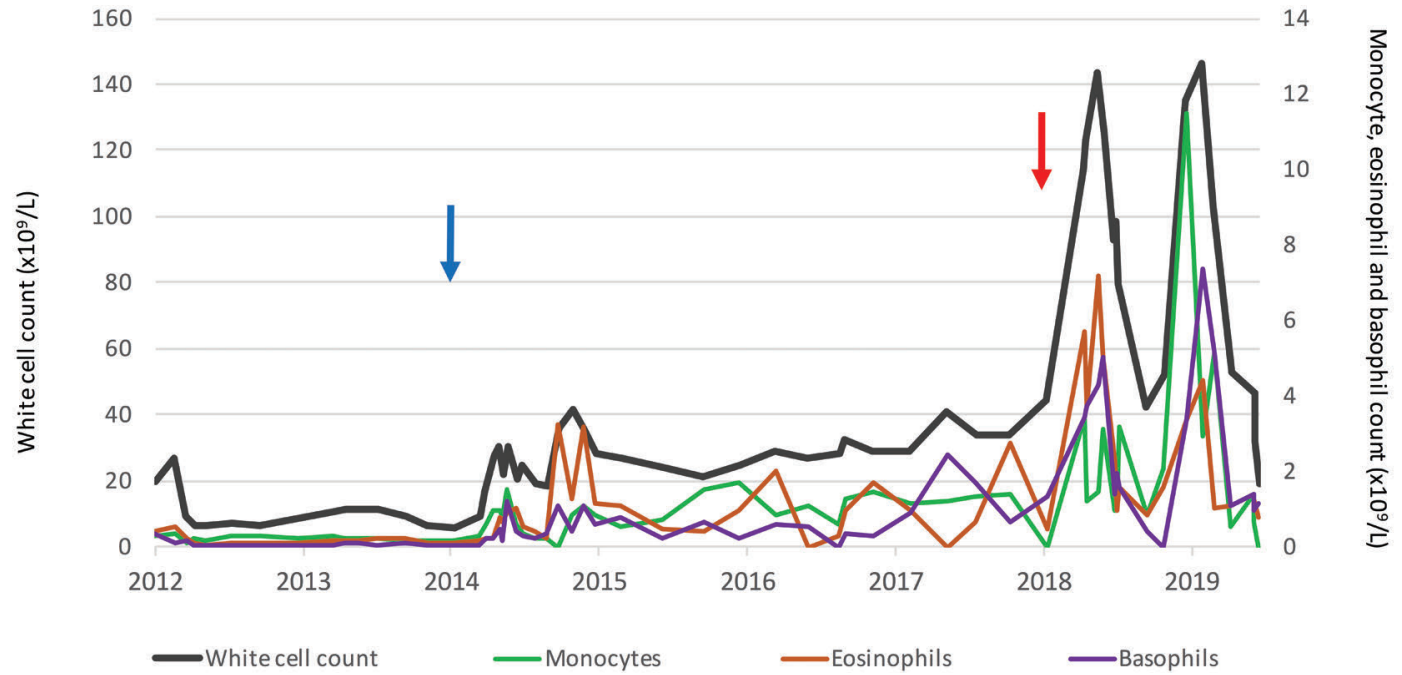

Figure 1. Change in total white cell count (primary y-axis), monocyte, eosinophil and basophil count (secondary y-axis) over time. Blue arrows indicate transformation to post-polycythemia myelofibrosis (PV-MF) with an associated increase in JAK2 p.V617F variant allele frequency (VAF) $=0.51-0.82$. Red arrows indicate transformation to accelerated phase PV-MF (with marked monocytosis, eosinophilia and basophilia), accompanied by a new MPL mutation: p.Y591D. The purple bar indicates treatment with ruxolitinib. The green bar indicates treatment with hydroxycarbamide.

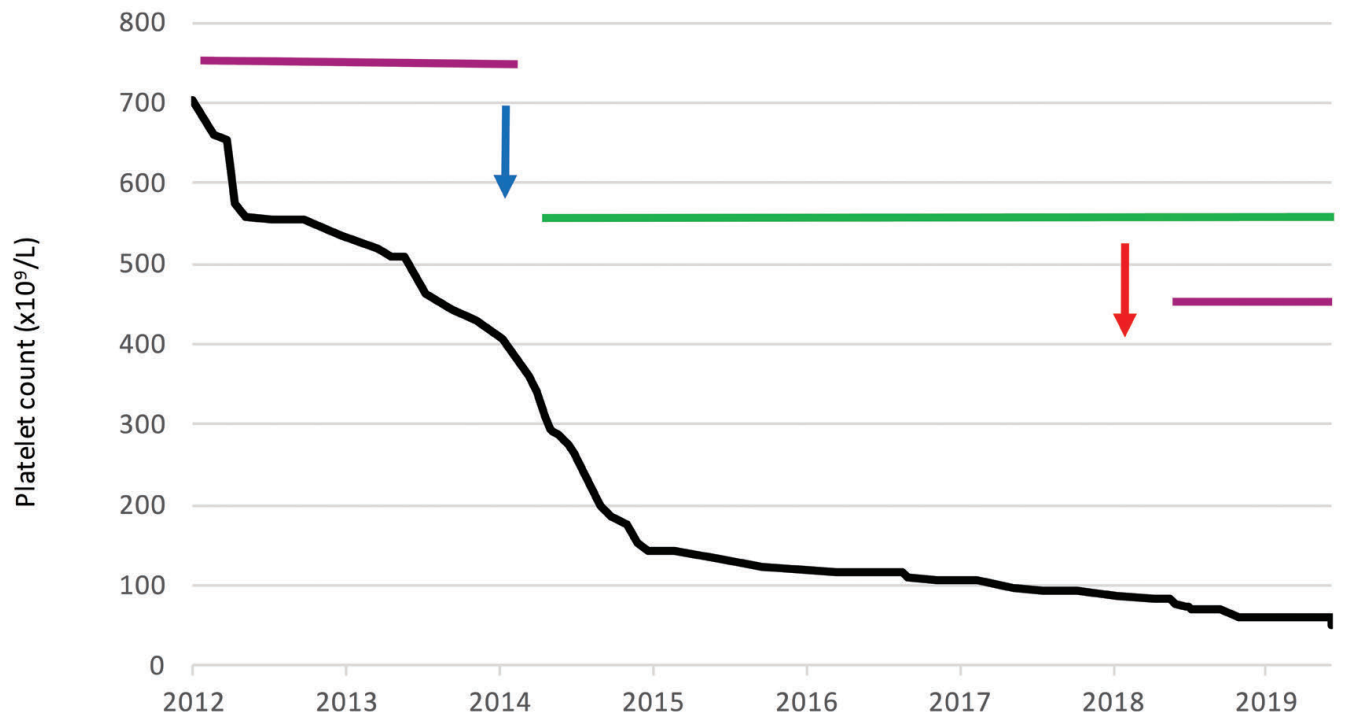

Figure 2. Change in platelet count over time. For the meaning of the arrows, see Figure 1. 


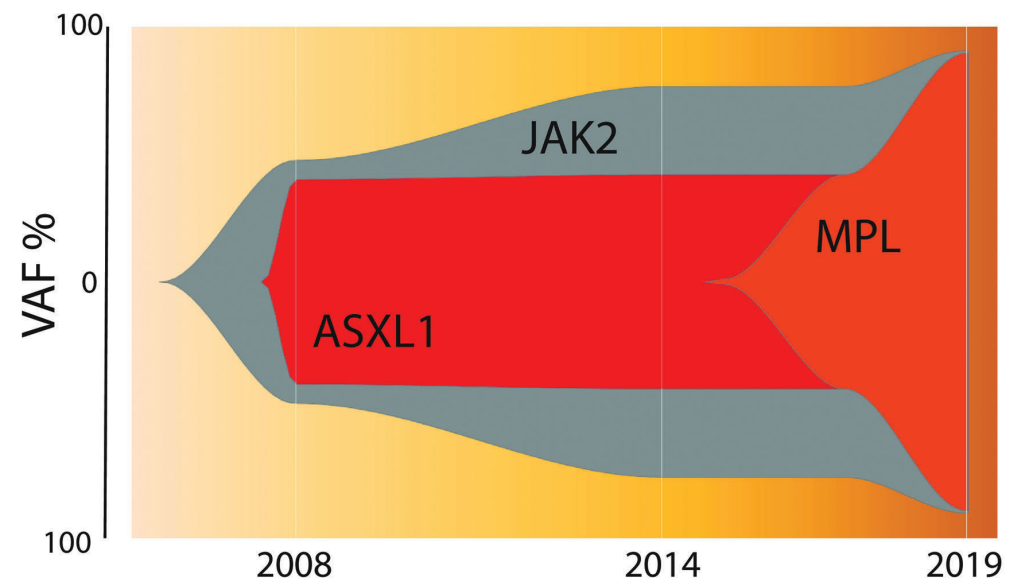

Figure 3. Clonal architecture over time. Visual representation of the variant allele frequency (VAF) for the three variants at the three time points with extrapolations as per the 'fishplot' package in R studio. ${ }^{15}$

so it is unlikely to be responsible for progression (Figure 3). The p.Y591D mutation affects 1 of 2 critical YRRL amino acid motifs in the cytoplasmic domain of MPL. ${ }^{10,11}$ It binds to the AP2 complex of clathrin-associated proteins and is critical for clathrin-mediated endocytosis of $M P L$, and thus downregulation of signalling following thrombopoietin (TPO) stimulation. ${ }^{12} \mathrm{Ba} / \mathrm{F} 3$ cells expressing MPL p.Y591D have a proliferative advantage, increased sensitivity to low doses of TPO, and increased JAK-STAT5 and ERK signalling compared to Ba/F3 cells expressing wild-type MPL. ${ }^{12,13}$ Although the acquisition of MPL p.Y591D was associated with the marked leucocytosis, we cannot be certain additional mutations in genes not represented in the comprehensive NGS panel contributed to progression.

Co-existence of driver mutations in $P h-\mathrm{MPN}$ is uncommon. It has generally been reported in patients with a low allelic burden of the JAK2 p.V617F mutation. ${ }^{3,4} \mathrm{~A}$ recent study of 164 patients with essential thrombocytosis (ET) reported co-existing CALR or MPL mutations in $4.9 \%$, all occurring in patients with a $J A K 2$ p.V617F allelic burden of $<4 \%{ }^{4}$ The patients with co-existing driver mutations had a higher platelet count than those with a single driver mutation, however, further implications of the co-existing driver mutations remain unclear.

The true incidence of MPL p.Y591D mutation in MPN patients is unknown. It is encoded by exon 11 of $M P L$, and therefore it is not covered by most NGS panels. It was previously discovered in triple negative ET and MF patients following whole exome sequencing, ${ }^{10,13}$ and was found in this case because our panel covers the entire coding region of MPL. ${ }^{8}$ Thus, the true prevalence of noncanonical MPL mutations in MPN is likely under-recognised. Furthermore, co-inheritance of MPL p.Y591D with JAK2 p.V617F may occur during accelerated disease more commonly than currently appreciated.

Two cases of co-existing JAK2 p.V617F and MPL p.Y591D have been previously reported. Feenstra et al. reported a case of ET that was initially diagnosed as a triple-negative MPN. ${ }^{13}$ Five years after diagnosis, the patient acquired a $J A K 2$ p.V617F mutation. The entire coding region of $M P L$ was retrospectively sequenced by Sanger, detecting the p.Y591D mutation. Kawamata et al. reported the other case in a patient with PV. ${ }^{11}$ No clinical data was reported for either of these two patients. In contrast, our patient with post-PV MF had JAK2 p.V617F and ASXL1 p. R1415Ter for 5 years prior to acquisition of the MPL p.Y591D mutation (Figure 3). The MPL mutation was associated with UPD at chromosome 1, and an accelerated disease course with marked leucocytosis and splenomegaly without evidence of transformation to acute myelogenous leukemia (AML).

Comprehensive genomic analysis by NGS plays an important role in the diagnosis and prognosis of $P h-M P N .{ }^{5,10,14}$ With routine and sequential use at times of alterations in clinical parameters, it can help determine the existence of additional mutations to the original driver mutations. Integration of mutational characteristics with the clinical disease course is essential to predict an individual patient's outcomes and help support their treatment. Our case is unusual in that a rare mutation in $M P L$ was responsible for rapid acceleration of JAK2 p.V617F driven PV-MF, and apparent 'failure' of ruxolitinib but subsequent re-establishment of disease control with addition of hydroxycarbamide.

\section{Jeremy Ong, ${ }^{1,2}$ Jane I. Lin, ${ }^{1}$ Helen Mitchell, ${ }^{3}$}

Susan Morgan ${ }^{1,2 \#}$ and Andrew C. Perkins ${ }^{1,2,3 \#}$

\#SM and ACP contributed equally as co-senior authors

${ }^{1}$ Department of Pathology, Alfred Health; ${ }^{2}$ Department of Clinical Haematology, Alfred Health and ${ }^{3}$ Australian Centre for Blood

Diseases, Monash University, Melbourne, Australia

Correspondence: ANDREW PERKINS

andrew.perkins@monash.edu

doi:10.3324/haematol.2020.250969

Information on authorship, contributions, and financial \& other disclosures was provided by the authors and is available with the online version of this article at www. haematologica.org.

\section{References}

1. Arber DA, Orazi A, Hasserjian R, et al. The 2016 revision to the World Health Organization classification of myeloid neoplasms and acute leukemia. Blood. 2016;127(20):2391-2405.

2. De Roeck L, Michaux L, Debackere K, Lierman E, Vandenberghe P, Devos T. Coexisting driver mutations in MPN: clinical and molecular characteristics of a series of 11 patients. Hematology. 2018;23(10):785-792.

3. Nussenzveig RH, Pham HT, Perkins SL, Prchal JT, Agarwal AM, Salama ME. Increased frequency of co-existing JAK2 exon-12 or MPL exon-10 mutations in patients with low JAK2(V617F) allelic burden. Leuk Lymphoma. 2016;57(6):1429-1435.

4. Usseglio F, Beaufils N, Calleja A, Raynaud S, Gabert J. Detection of CALR and MPL mutations in low allelic burden JAK2 V617F essential thrombocythemia. J Mol Diagn. 2017;19(1):92-98.

5. Vannucchi AM, Lasho TL, Guglielmelli P, et al. Mutations and prognosis in primary myelofibrosis. Leukemia. 2013;27(9):1861-1869.

6. Tefferi A, Lasho TL, Guglielmelli P, et al. Targeted deep sequencing 


\section{Case Reports}

in polycythemia vera and essential thrombocythemia. Blood Adv. 2016;1(1):21-30.

7. Tefferi A, Vannucchi AM. Genetic risk assessment in myeloproliferative neoplasms. Mayo Clin Proc. 2017;92(8):1283-1290.

8. Magor GW, Tallack MR, Klose NM, et al. Rapid molecular profiling of myeloproliferative neoplasms using targeted exon resequencing of 86 genes involved in JAK-STAT signaling and epigenetic regulation. J Mol Diagn. 2016 9/1;18(5):707-718.

9. Tate JG, Bamford S, Jubb HC, et al. COSMIC: the catalogue of somatic mutations in cancer. Nucleic Acids Res. 2019;47(D1):D941D947.

10. Grinfeld J, Nangalia J, Baxter EJ, et al. Classification and personalized prognosis in myeloproliferative neoplasms. N Engl J Med. 2018;379(15):1416-1430
11. Kawamata N, Ogawa S, Yamamoto G, et al. Genetic profiling of myeloproliferative disorders by single-nucleotide polymorphism oligonucleotide microarray. Exp Hematol. 2008;36(11):1471-1479.

12. Hitchcock IS, Chen MM, King JR, Kaushansky K. YRRL motifs in the cytoplasmic domain of the thrombopoietin receptor regulate receptor internalization and degradation. Blood. 2008;112(6):2222-2231.

13. Milosevic Feenstra JD, Nivarthi $\mathrm{H}$, Gisslinger $\mathrm{H}$, et al. Whole-exome sequencing identifies novel MPL and JAK2 mutations in triple-negative myeloproliferative neoplasms. Blood. 2016;127(3):325-332.

14. McClure RF, Ewalt MD, Crow J, et al. Clinical significance of DNA variants in chronic myeloid neoplasms: a report of the Association for Molecular Pathology. J Mol Diagn. 2018;20(6):717-737.

15. Miller CA, McMichael J, Dang HX, et al. Visualizing tumor evolution with the fishplot package for R. BMC Genomics. 2016;17(1):880. 